\title{
Recombinant human MMP-2 associated with monoolein improves bone repair
}

\author{
de Figueiredo FAT ${ }^{1}$, Azevedo $\mathrm{JP}^{2}$, Lara $\mathrm{MG}^{3}$, Meschiari $\mathrm{CA}^{4}$, Issa $\mathrm{JPM}^{2}$, Gerlach $\mathrm{RF}^{2}$, Lamano $\mathrm{TLC}^{2}$ \\ Department of Dental Materials and Prosthodontics, School of Dentistry of Ribeirao Preto, \\ University of Sao Paulo, Brazil. fellipe.figueiredo@usp.br
}

\section{ABSTRACT}

OBJECTIVE: The main objective of the present study was to investigate the possible osteostimulatory action of recombinant human matrix metalloproteinase-2 (rhMMP-2) implanted in a bone defect made in calvaria of rats, bounded to the monoolein as carrier.

METHODS: Forty-four adult male Wistar rats (about $600 \mathrm{~g}$ body weight) underwent surgery in order to create a spherical defect in parietal bone on the right side of the median sagittal suture by using $4 \mathrm{~mm}$ diameter of a trephine drill. Animals were divided into three groups: no treatment (control, C), treatment with rhMMP-2 diluted in monoolein liquid crystal (rhMMP-2) and negative control with monoolein (M). The groups were divided into two experimental times, 2- and 4-weeks of experimental time.

RESULTS: The rate of new-formed bone, estimated by the number of points on new-formed cancellous bone, was in enhanced rhMMP-2 group in both periods in comparison to $\mathrm{C}$ or $\mathrm{M}$ groups.

CONCLUSION: There was no difference in bone neoformation between second to fourth week within groups. In the present study, monoolein alone had a negative role in the post-operative surgery, but monoolein associated with +rhMMP-2 had a positive role on releasing rhMMP-2 and enhance the rate of new-formed bone (Tab. 1, Fig. 5, Ref. 71). Text in PDF www.elis.sk

KEY WORDS: bone, bone defect, monoolein, rhMMP-2, biomaterial.

\section{Introduction}

Bone defects may result from congenital disorders, trauma, or others, and they are still a challenge for medical and dental treatment $(1,2)$. A variety of organic and synthetic biomaterials are available on the market, which differ by the origin, method of preparation, chemical composition, structural characteristics and thus the properties and response evoked in biological tissues (2). Tricalcium phosphate/hydroxyapatite (3), fibrin selant (4), fribin (5), calcium sulphate (6) decellularized bovine bone scaffolds (7), hydrogel based on elastin (8) and gelfoam surgical sponges (9) are examples of biomaterials used in bone engineering. Despite scientific and technological advances in this area, there is still no material that meets all the requirements to ensure the success of the treatment.

${ }^{1}$ Department of Dental Materials and Prothesis, School of Dentistry of Ribeirao Preto, University of Sao Paulo - Av. Cafe S/N, Ribeirao Preto, SP, Brazil, ${ }^{2}$ Department of Basic and Oral Biology, School of Dentistry of Ribeirao Preto, University of Sao Paulo - Av. Cafe S/N, Ribeirao Preto, SP, Brazil, ${ }^{3}$ Department of Pharmaceutical Sciences, School of Pharmaceutical Sciences of Ribeirao Preto, University of Sao Paulo - Av. Cafe S/N, Ribeirao Preto, SP, Brazil, and ${ }^{4}$ Department of Physiology, School of Medicine of Ribeirao Preto, University of Sao Paulo - Av. dos Bandeirantes 3900, Ribeirao Preto, SP, Brazil

Address for correspondence: F.A.T. de Figueiredo, Prof, Department of Dental Materials and Prosthodontics, School of Dentistry of Ribeirao Preto, University of Sao Paulo, Av. Cafe S/N-Ribeirao Preto, São Paulo, Brazil. CEP: 14040-904

Phone: +55.16 .33154008$
Matrix metalloproteinases (MMPs) are enzymes that regulate the composition of the extracellular matrix by cleaving matrix components, facilitating tissue remodeling and cell migration. There is an association between increased activity of several MMPs and bone loss in several pathological situations (10-12). Recent evidence, however, questions the essentially "degrading" bone role of MMPs, particularly matrix metalloproteinase-2 (MMP-2), which exhibits high levels of activity in osteogenic cell culture (13) and has increased expression in the early events of implant osseointegration (14). A mutation in MMP-2 gene inactivates proteolytic activity resulting in severe osteolytic phenotype in adults $(15,16)$, which demonstrates the importance of MMP-2 in bone reabsorption but also in bone formation.

MMPs, such as the collagenases-A (MMP-2), have a primordial role in bone remodeling capacity of active nonmineralized osteoid $(17,18)$. Osteoblasts can express MMPs, including MMP-2 and, osteoclasts can also express MMP-2 (19). So, the presence of an active MMP-2 at bone repair sites can have a positive effect on the final bone repair. The absence of MMP-2 may be linked to lower mineral density and higher bone porosity, which lead to a weaken bone (19). The absence of MMP has been linked with fractures and multicentric osteolysis, which is well known on the mutations of MMP-2 coding (20).

Monoolein is a water-soluble molecule (polar lipid) (21) that has a very low toxic biological property (22) with the capacity to be used in controlled release formulations in pharmaceuticals, food and cosmetics $(23,24)$. The association between the monoolein 
as a well-known carrier and rhMMP-2 could have better results on bone repair.

Based on these properties, the main objective of the present study was to evaluated the association between MMP-2 and monoolein-carrier, which could enhance the rate of newformed bone.

\section{Materials and methods}

\section{Biomaterials}

The rhMMP-2 was produced by genetically modified bacteria (Escherichia coli strain BL21-PLys) by recombinant DNA technology. The rhMMP-2 protein was produced in our laboratory and specific details on its production as well as enzymatic activity data were described in a previously published study (25). Additionally, a purification step was performed in order to remove lipopolysaccharides (LPS) from rhMMP-2 samples. Samples were mixed with triton $\mathrm{x} 100$ and Centrifuged for 10 minutes and the supernatant was collated. The LPS concentration in rhMMP-2 solution was determined by the kit QCL-100 (50647-U, Lonza do Brasil, Especialidades Químicas, Sao Paulo, Brasil). Samples of rhMMP-2 containing concentrations lower than $0.4 \mathrm{EU} / \mu \mathrm{g}$ of protein (endotoxin units) which is suitable for parenteral use (26). The rhMMP-2 samples were lyophilized and stored at $-80^{\circ} \mathrm{C}$ up to use.

Monoolein used in the present work was a classical monoglyceride derivate from canola oil (Myverol 18-99, Eastman Chemical Company, Tennessee, United States) (27).

\section{Releasing assay}

The present assay was assed to ensure how much rhMMP-2 was released and how much rhMMP-2 was need to be used in the monoolein vehicle. The rhMMP-2 samples were resuspended in phosphate saline buffer ( $\mathrm{PBS}, \mathrm{pH}=7.4$ ) and sterilized by $0.2 \mu \mathrm{m}$ filtration. Monoolein was melted in water-bath at $47{ }^{\circ} \mathrm{C}$ and mixed with rhMMP-2 PBS solution in 3:2 ratio. Cellulose in monoolein-carrier was used as a negative control. The rhMMP-2 in monoolein-carrier samples were transferred to holder, sealed and incubed at room temperature in order to change the monoolein in to cubic phase (28). Holders were attached to micro pump with $500 \mu \mathrm{L} / \mathrm{h}$ flow rate of PBS (pH 7.4) with $1 \mathrm{mmol} / \mathrm{L}$ of EDTA, an MMP inhibitor, in order to prevent autolysis (29). Equated samples were collected at 1, 2, 4 and 24h, lyophilized and rhMMP-2 concentration was assessed by enzyme-linked immunosorbent assay (ELISA).

On the results part, the authors discussed the results of the releasing assay, the main concentration of $12.5 \mu \mathrm{g}$ of rhMMP-2 was used in association with monoolein.

\section{Animals and surgical procedure}

This study was approved by the University of Sao Paulo, School of dentistry of Ribeirao Preto - Committee on Ethics in Animal Use (protocol number 10.1.1305.53.2) which follows the international standards for animal use and management. Fortyfour adult male Wistar rats [(about $600 \mathrm{~g}$ body weight (bw)] were kept at room temperature in light-controlled environment with a 12:12-h light-dark cycle and had free access to water and standard mouse chow. This study was approved by the Committee on Ethics in Animal Use (Process No. 10.1.1305.53.2).

Animals were weighed and anesthetized with a $10 \% \mathrm{Ke}-$ tamine Hydrochloride solution (Ketamine Agener, Uniao Quimica Farmaceutica Nacional S / A, Embu-Guaçu, SP, Brazil, 75 mg/kg) associated with Xylazine (Dopaser, Laboratorios Calier S/A, Barcelona, Spain, $10 \mathrm{mg} / \mathrm{kg}$ intraperitoneally) at the dose of $0.2 \mathrm{ml}$ solution/100 g bw. After anesthesia, with the animal placed in a ventral decubitus position and incision of the cutaneous and muscular tissues was done approximately $3 \mathrm{~cm}$ long, anteriorly-posteriorly, in the central region of the calvaria, using a scalpel blade.

The soft tissues were dived with scalloped scissors for exposure to the periosteum, and then carefully debulked to expose the desired bone surface within a sterile surgical field. A spherical bone defect was created in the parietal bone, on the right side of the median sagittal suture, using $4 \mathrm{~mm}$ diameter of a trephine drill (Trephine 4 mm MK Dent, Bargteheide, Germany) fitted with a micromotor (Kavo, Jaguaçu, Santa Catarina, Brazil). The bone board was completely perforated, maintaining the integrity of the meninges and creating a cylindrical defect of $4 \mathrm{~mm}$ in diameter and about $1.5 \mathrm{~mm}$ in thickness. The entire surgical procedure was perform under constant and abundant irrigation with $0.9 \%$ sterile saline solution $(30,31)$. The bone defect was maintained with the natural clotting of the blood clot or with a gel-like biomaterial (monoolein or metalloproteinase bound to monoolein) using a sterile spatula and filling the defect completely, but avoiding extravasation (about $75 \mathrm{mg}$ of material by bone defect). Subsequently, the periosteum was folded and sutured with catgut 5-0 thread (Ethicon, Johnson \& Johnson, São José dos Campos, SP, Brazil) and the cutaneous tissue sutured with silk thread 4.0 (Ethicon, Johnson \& Johnson, São José dos Campos, SP, Brazil). After surgery, the animals received a single dose $(0.2 \mathrm{~mL} / 100 \mathrm{~g}$ of body weight, intramuscular) of veterinary antibiotic solution for small animals (Fort Dodge, Campinas, Sao Paulo, Brazil) and remained under observation for 3 hours.

The groups were divided into 3 major groups and these groups were subdivided into 2 periods:

Control group: bone defects were maintained with natural clotting of the blood clot and the animals were sacrificed after 2 $(\mathrm{n}=9)$ and 4 weeks $(\mathrm{n}=9)$;

Monoolein group: the bone defects were filled with monoolein (carrier) and the animals were sacrificed after $2(\mathrm{n}=3)^{*}$ and 4 weeks $(\mathrm{n}=7)^{*}$;

Monoolein $+M M P-2$ group: the bone defects were filled with monoolein-bound to $12.5 \mu \mathrm{g}$ of rhMMP-2 and the animals were sacrificed after $2(n=7) *$ and 4 weeks $(n=9)$.

*The difference between the " $n$ " is due to the death of the animals during the surgery and due to the exudate present in the defect just after the surgery, showing that the monoolein alone could be dangerous for critical osteo-defect on calvaria. The most affected group was the Monoolein group 2-weeks, but the statistical analysis was not prejudicated.

\section{Histological processing and analysis}

In the 2- and 4-week periods after surgery, the animals received an overdose of anesthetic $(0.4 \mathrm{~mL} / 100 \mathrm{~g}$ body weight, intraperito- 

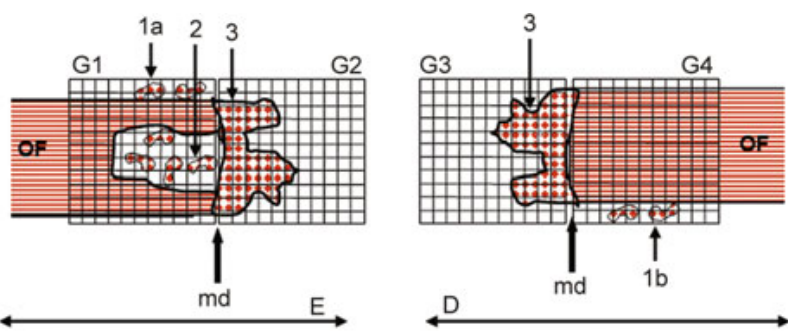

Fig. 1. Schematic representation of the left $(\mathrm{L})$ and right $(\mathrm{R})$ borders of histological section of bone defect showing newformed cancellous bone in the subperiosteal area $(1 \mathrm{a}, 1 \mathrm{~b})$, by internal remodeling of the frontal bone (2) and advancing centripetally inside the defect (3). OF = frontal bone; $\mathbf{m d}=$ defect $\operatorname{margin} ; \mathbf{G 1}, \mathbf{G 2}, \mathbf{G 3}, \mathbf{G 4}=$ grids for differential point counting (points are represented by the intersection of perpendicular lines, 22 horizontal lines. and 29 vertical lines).

neal route) and were decapitated. After surgical removal, the calvaria was fixed by immersion in $10 \%$ formaldehyde solution for $48 \mathrm{~h}$, decalcified (for 6 days in $20 \%$ sodium citrate solution and $30 \%$ formic acid, changing the solution every $48 \mathrm{~h}$ ) and washed
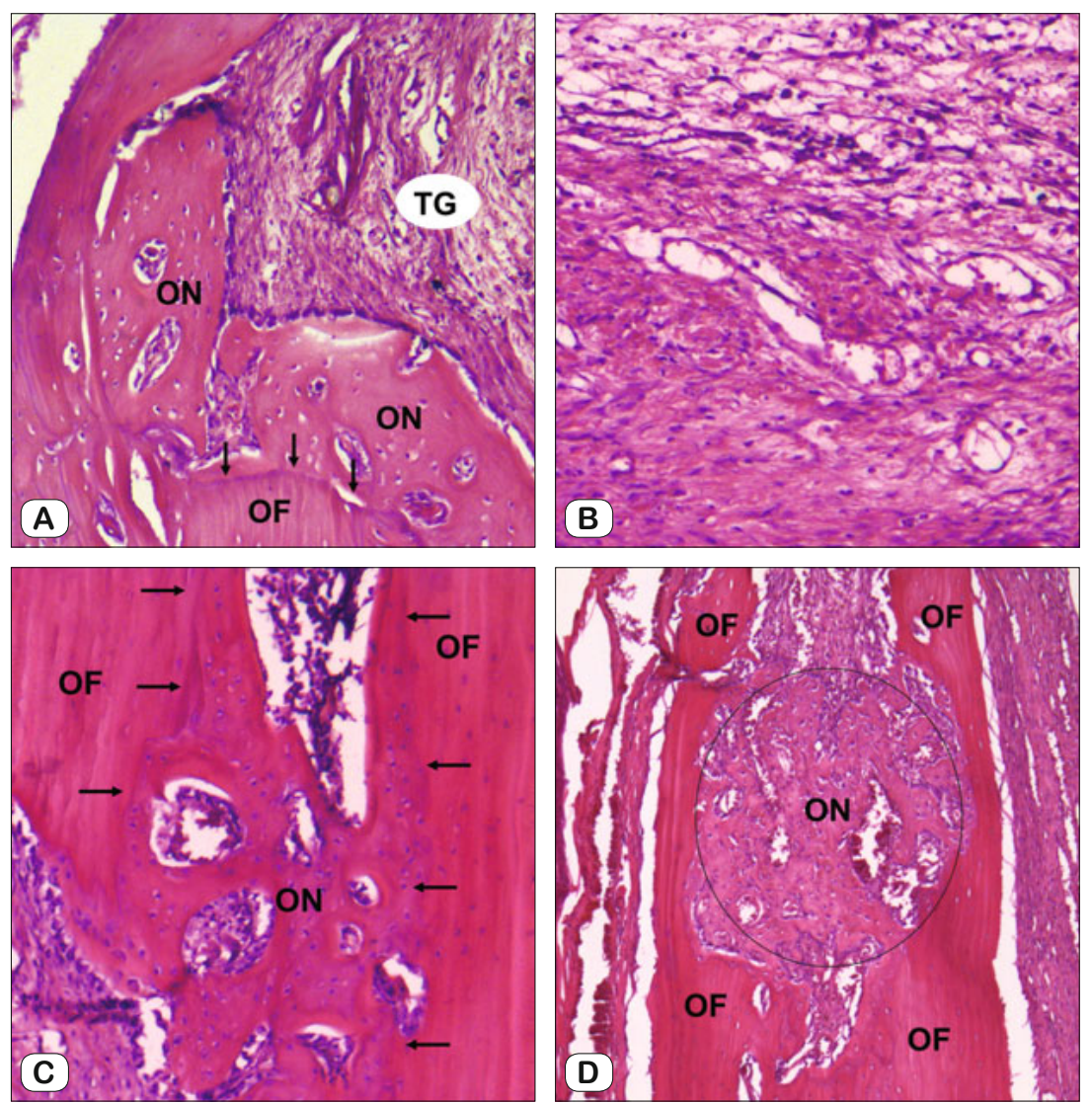

Fig. 2. Histological appearance of bone defect in the Control Group of animals - 2 weeks (A). Newformed cancellous bone (ON) from the frontal bone (OF) of the defect margin (arrows point to the cementitious line between the two), TG = granulation tissue. (B) Detail of the bone defect filling granulation tissue. Histological aspect of bone defect of the Control Group - 4 weeks, showing areas of new bone cancellous $(\mathrm{ON})$ forming by internal remodeling of the frontal bone (OF), marked by the arrows in (C) and by the circle in (D). Staining with hematoxylin eosin, (C) objective $10 \mathrm{X}$, (D) objective $5 \mathrm{X}$.
Tab. 1. Release test of Monoolein by hours.

\begin{tabular}{lcccccc}
\hline \multirow{2}{*}{ Initial mass } & \multicolumn{5}{c}{ Collecting time (in hours) } & Total mass \\
\cline { 2 - 6 } & $1 \mathrm{~h}$ & $2 \mathrm{~h}$ & $3 \mathrm{~h}$ & $4 \mathrm{~h}$ & $24 \mathrm{~h}$ & released \\
\hline $37.5 \mu \mathrm{g}$ & 0 & 1.1 & 8 & 0 & 0 & 9.1 \\
$19 \mu \mathrm{g}$ & 0 & 2.9 & 8 & 0 & 0 & 8.9 \\
$3.75 \mu \mathrm{g}$ & 0 & 0.7 & 0.6 & 3.4 & 0 & 1.3 \\
$37.5 \mu \mathrm{g}+$ & 0 & 0 & 0 & 0 & 0 & 0
\end{tabular}

*Cellulose is a vehicle that does not interfere with the release of the protein, in the present study it was used as control

in running water for $24 \mathrm{~h}$. The pieces were dehydrated, diaphanized and embedded in paraffin, oriented to allow longitudinal semi-serial cuts of $6 \mu \mathrm{m}$ thickness (using 1 each 11 cuts), starting with the median lateral edge of the defect. The slides were stained with hematoxylin and eosin solutions.

Histological examination of all cuts present on all slides of each animal was performed. For histomorphometry, $4.5 \pm 0.3$ (mean \pm standard error of the mean) histological sections were selected per animal.

Optical microscope (Zeiss Axiostar Plus, Gottingen, Germany) equipped with a digital video camera for image capture (Zeiss Axiocam IC, Gottingen, Germany) and a software of public domain (Image J, version 1.38 , available at www.mcbiophotonics.ca/ imagej) was used for the quantification of the newformed bone. Among the alternatives offered by the histometric program, a differential score of points was used, using a grid of 638 points equidistant (intersection of 22 by 29 lines) that overlapped the different components of filling the bone defect in repair (tissue granulation, newformed cancellous bone, medullary connective tissue). It is considered that the number of points counted on each component is proportional to the relative volume occupied by it.

In each selected histological section 4 grids were placed, two on the left border of the histological image of the defect (one from the margin of the defect towards its interior and one from the margin of the defect towards the contiguous frontal bone) and two at the right border, obeying the same criterion (Fig. 1).

In this study, $4798.2 \pm 272.9$ points counted (average \pm standard error of the mean) per animal (only those on the repair components) and the volume of newformed cancellous bone was estimated by the number of points counted on them. In the images used to estimate bone neoformation, measurements of defect extension were also performed (32).

\section{Statistical analysis}

The comparison among the 3 groups was perform by the non-parametric test of 


\section{$571-579$}

Kruskal-Wallis for multiple comparison of independent samples, followed by the Mann-Whitney test for comparison of samples 1 to 1, using the GraphPad Prism 5 (GraphPad, San Diego, California, United States).

\section{Results}

\section{Releasing assay}

The releasing assay is very important to know how does monoolein interact with the active rhMMP-2, this assay gave to the research group the maximum release with lower doses of rhMMP-2.

Concentrations of rhMMP-2 associated with monoolein were determined by ELISA, the releasing results are shown in supplemental Table 1 . In the present study it was not possible to quantify the release of 1 hour in any assays, it can infer that the rhMMP-2 associated with monoolein have a low release, which is a promisor finding. For example: *Cellulose associated with $37.5 \mu \mathrm{g}$ of rh MMP-2 had no release at 2 hours, and most likely, released all the content before 1 hour. Monoolein associated with rhMMP-2
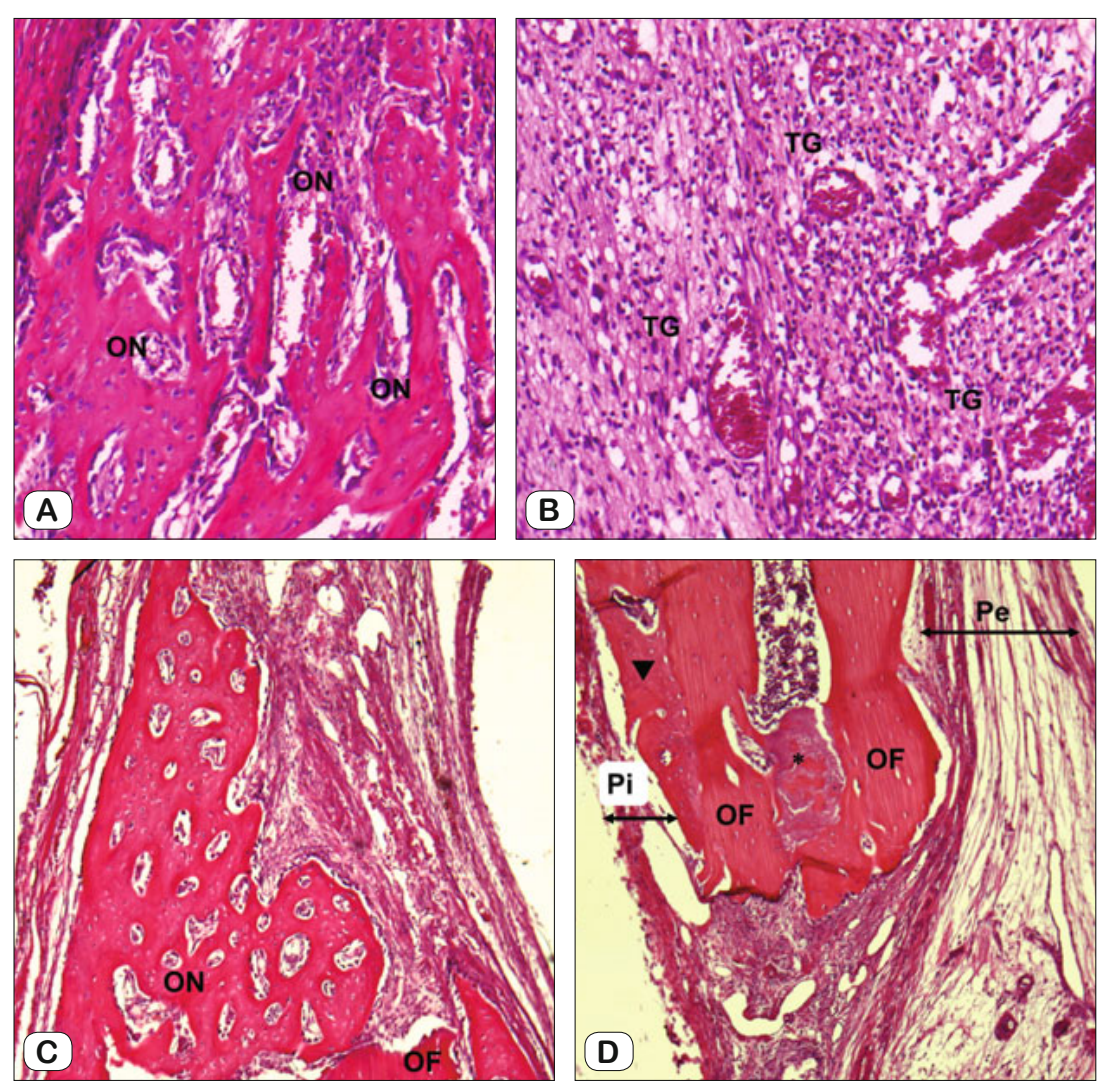

Fig. 3. Histological appearance of bone defect of Monoolein Group animal - 2 weeks (A). Newformed cancellous bone (ON) from the frontal bone of the margin of the defect (A) and richly vascularized granulation tissue (TG) exhibiting lymphoplasmacytic inflammatory infiltrate (B). Staining with hematoxylin eosin, objective $10 \mathrm{X}$. Histological aspect of bone defect of Monolein Group animal - 4 weeks. (C) New cancellous bone $(\mathrm{ON})$ forming from the frontal bone (OF) of the margin of the defect. (D) Internal remodeling of the frontal bone (OF) near the border of the defect $(\square)$ and new bone formation with internal subperiosteal (Upside-down arrow); the arrows mark the internal $(\mathrm{Pi})$ and outer $(\mathrm{Pe})$ periosteal laminae. Staining with hematoxylin eosin, objective $5 \mathrm{X}$. causes low releasing of the protein and might prevent the monoolein to recruit some dangerous inflammatory cells that lead to death of the animals due tothe surgery.

In the present study the releasing assay of $37.5 \mu \mathrm{g}$ of rhMMP-2 ing-power of rhMMP-2 has a promising result on the bone repair. The concentration of $12.5 \mu \mathrm{g}$ of rhMMP- 2 was used in the present study and it was expected that a release between $25 \%$ and $50 \%$

\section{Qualitative analyses}

Control group

The findings referenced by the control group are shown in Figure 2. Figure 2A Demonstrates a bone repair at 2 weeks. Newformed cancellous bone $(\mathrm{ON})$ from the frontal bone $(\mathrm{OF})$ of the defect margin (arrows point to the cementitious line between the two), $\mathrm{TG}=$ granulation tissue (Figure $2 \mathrm{~A})$. Figure 2B demonstrates details of the bone defect filling granulation tissue. The histological aspects of bone defect of the control group by 4 weeks are demonstrated by the areas of newformed cancellous bone $(\mathrm{ON})$ formed by internal remodeling of the frontal bone (OF), marked by the arrows in (Figure $2 \mathrm{C}$ ) and by the circle in Figure 2D. At the end of the second week, the defect of the control animals was predominantly filled by highly vascularized granulation tissue with collagen fibrils and chronic inflammatory infiltrate (lymphoplasmocytes). From the margins of the defect, there was centripetal neoformation of primary immature bone (without lamellar organization), with irregular cancellous structure surrounded by osteoblasts and matrix imprisoning large numbers of osteocytes. While in some animals the bone neoformation was incipient, in others it was more prominent, with irregular cancellous tissue advancing into the granulation tissue.

\section{Monoolein group}

The histological examination of the defect of the animals of the monoolein group showed no significant difference compared to the control animals of the same period (comparing the Figure 2 vs Figure 3). The findings of the group Monoolein are demonstrated in Figure 3. By 2 weeks (Figure 3A), newformed cancellous bone $(\mathrm{ON})$ from the frontal bone of the margin of the defect and richly vascularized granulation tissue (TG) 

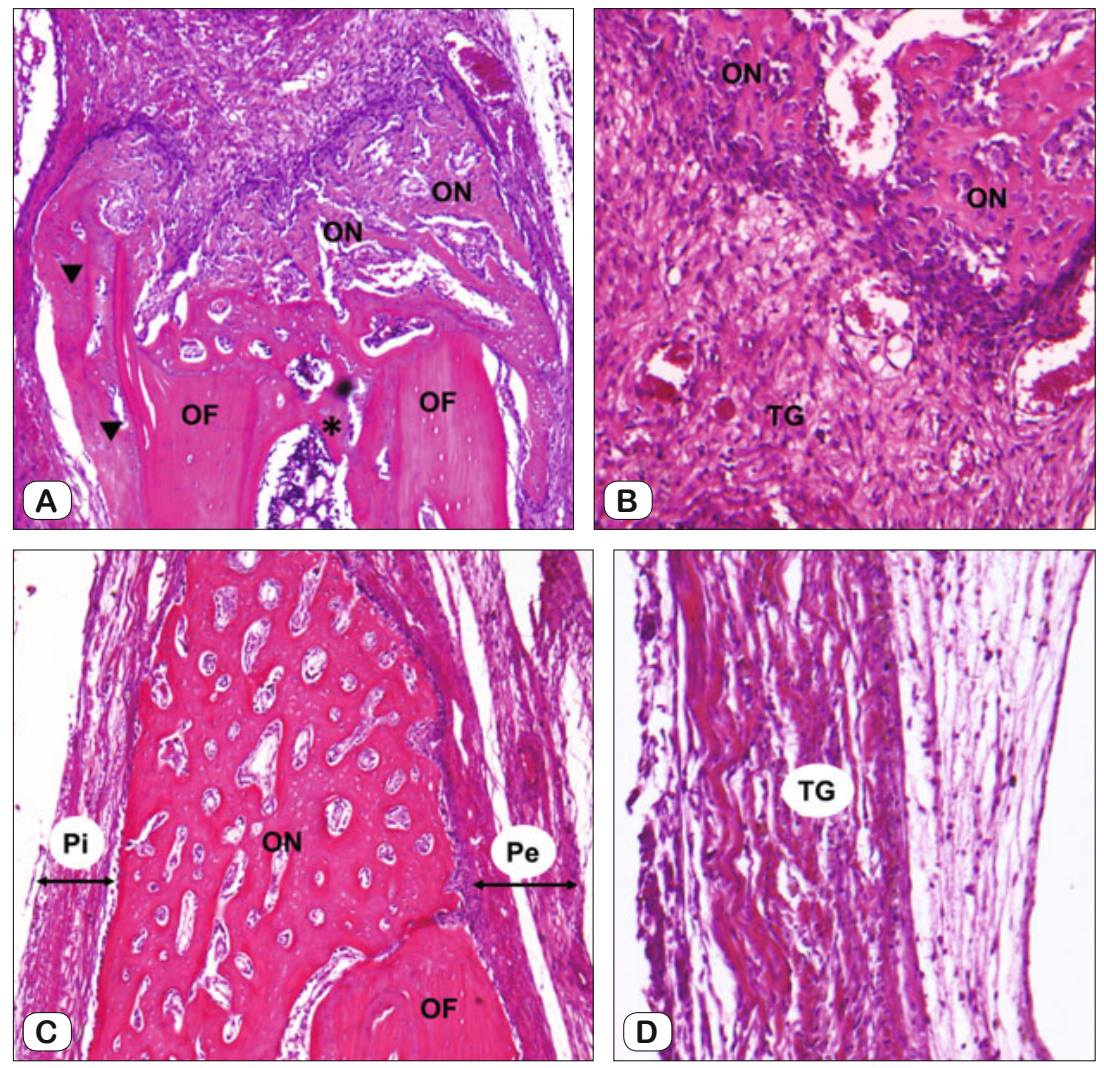

Fig. 4. Histological appearance of animal bone defect of MMP-2 group - 2 weeks. (A) Centripetal bone neoformation from the frontal bone (OF) of the margin of the defect, as well as internal remodeling of the frontal bone $(\square)$ and internal subperiosteal (upside-down arrow) localization. (B) Newformed cancellous bone (ON) and granulation tissue (TG) filling the bone defect. Staining with hematoxylin eosin, (A) objective $5 \mathrm{X}$, (B) objective $10 \mathrm{X}$. Histological aspect of bone defect of animals of Group MMP-2-4 weeks. (C) Bone neoformation (ON) prominent from the frontal bone (OF) of the margin of the defect; the arrows mark the internal (Pi) and outer (Pe) periosteal laminae. (D) Connective tissue (TG) of the bone defect exhibiting lymphoplasmacytic inflammatory infiltrate. Staining with hematoxylin eosin, objective $5 \mathrm{X}$.

of the defect; the arrows mark the internal $(\mathrm{Pi})$ and outer $(\mathrm{Pe})$ periosteal laminae. Connective tissue (TG) of the bone defect exhibiting lymphoplasmacytic inflammatory infiltrate are demonstrate in figure 4D. The repair of the present animals generally followed the same pattern described for controls, with defects predominantly filled by highly vascularized granulation tissue and with variable intensity of lymphoplasmacytic inflammatory infiltrate exhibiting centripetal bone neoformation of primary immature bone with no apparent progress from the second to the fourth week after surgery. A peculiar finding of this group was the marked bone neoformation in the vicinity of the defect, both under the internal and/or external laminae of the periosteum and through internal remodeling of the frontal bone (Fig. 4).

The histological examination suggests a higher amount of newformed cancellous bone than in the control animals of the same period.

\section{Histomorphometric analysis}

Semi-serial histological sections $6 \mu \mathrm{m}$ thick (taking advantage of 1 in 11 cuts), starting with the medial lateral border of the defect. Thus, as the cuts deepened towards the central area, defects were obtained with increasing extent.

The rate of newformed bone, estimated by the number of points on newformed cancellous bone, was similar in the Control and Monoolein groups and significantly

exhibiting lymphoplasmacytic inflammatory infiltrate were present (Figure 3B). By 4 weeks, (Figure 3C) new cancellous bone (ON) forming from the frontal bone (OF) of the margin of the defect was present. Figure 3D shows internal remodeling of the frontal bone (OF) near the border of the defect $(*)$ and new bone formation with internal subperiosteal (Upside-down arrow); the arrows mark the internal $(\mathrm{Pi})$ and outer $(\mathrm{Pe})$ periosteal laminae.

\section{MMP-2 bound to Monoolein Group}

Figure 4 demonstrates the histological appearance of animal bone defect of MMP-2 bound to monoolein group at 2 weeks. Figure 4A shows the centripetal bone neoformation from the frontal bone (OF) of the margin of the defect, as well as internal remodeling of the frontal bone $(*)$ and internal subperiosteal (upside-down arrow) localization. Figure 4B shows newformed cancellous bone (ON) and granulation tissue (TG) filling the bone defect. Histological aspect of bone defect of animals in Group MMP-2 - 4 weeks. Figure 4C demonstrates bone neoformation $(\mathrm{ON})$ prominent from the frontal bone $(\mathrm{OF})$ of the margin higher in the MMP-2 bounded with monoolein animals, in both periods. There was no significant increase in bone neoformation from the second to the fourth week in any of the experimental groups (Fig. 5).

\section{Discussion}

Matrix Metalloproteinases have a promising potential in the clinical procedures and medical treatment as a biomarker for several diseases $(33,34)$. The critical defect made in calvaria of rodents is an in vivo model widely used to test the repair in several experimental situations for different strategies that aim to stimulate bone neoformation $(31,35,36)$. In the present study monoolein used as a carrier of rhMMP-2 released this protein slowly, helping this protein to act as inflammatory modulator of the repair of the bone defect. The major finding of the present study is that the presence of rhMMP-2 could attenuate the inflammatory cell recruited from the presence of monoolein, which was very beneficial for the final result of the bone formation. 


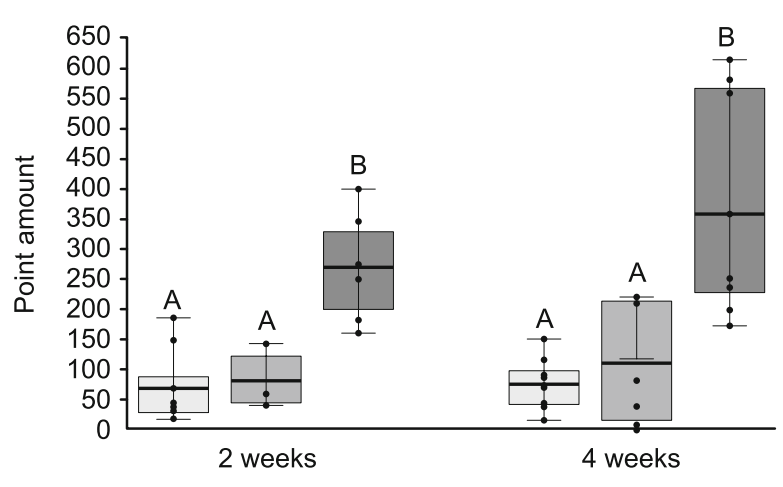

$\square$ Control $\square$ Monoolein $\square$ Monoolein + Metalloproteinase

Fig. 5. Median and interquartile range $(5 \%, 25 \%, 75 \%$ and $95 \%)$ referring to the amount of points on newformed cancellous bone in the Control, Monoolein and MMP-2 (metalloproteinase) groups, at 2 and 4 weeks (each point represents the measure of an animal). The Kruskal-Wallis nonparametric statistical test for multiple comparisons confirmed the difference among groups $(k=27, p=0.0001)$ and the Mann-Whitney test for comparison of samples 2 to 2 discriminating the difference of each sample, indicated in the figure with different letters $(A \neq B$, see table 1 for level of significance).

"Critical size" defect of bone was originally defined as one whose size, in a specific bone and in a particular animal species, would prevent spontaneous regeneration, even considering the whole life span $(35,37)$. However, most studies do not extend throughout animal life, more recently it has been proposed that "critical size" defects would be those whose size would prevent complete regeneration over the experimental period $(35,38)$. In contrast, subcritical size defects are complete spontaneous regeneration which is expected over a relatively short period of time (36).

In spite of the controversies regarding the size of calvaria „critical size“ defects of rats of different ages and varieties, the experimental pattern in adult rats has dimensions of the order of 8 $\mathrm{mm}$ in diameter (36). However, Cooper et al (2010) reported that even calvaria defects of significantly smaller rodents do not reach complete regeneration even over prolonged periods of time. The authors have observed that defects of $2.3 \mathrm{~mm}$ in rat calvaria presented regeneration in the order of $35 \%$ in 6 weeks (35).

In the present study, $4 \mathrm{~mm}$ diameter defects were made on the lateral to the median sagittal suture. The advantage of such a small defect is to avoid a part of the venous network of the dura mater. Additionally, the periosteum was incised and, after the defects were made, folded and sutured. The preservation of the periosteum is one of the important factors for the possibility of adequate bone regeneration $(36,39,40)$.

The presence of purulent (polymorphonuclear) exudates was $12 \%$ in the Control group, $37 \%$ in the Monoolein group and MMP2 bound with monoolein groups in the present study. Therefore, despite the aseptic care of the area and surgical materials, and disinfection of biomaterials, it was not possible to avoid some degree of contamination, which was higher in animals that had monoolein implanted alone. This result is already known in literature, when Monoolein is associated with a cationic liposome composed by surfactant dioctade-cyldimethylammonium bromide (DODAB), the monoolein plays a positive role on chain of fluidity of the DODAB (41) and our results suggests that rhMMP-2 modulates the inflammatory cells involved, the present modulations are wellknown from other studies $(42,43)$.

Lymphoplasmacytic infiltrate was more pronounced in most of the implanted animals, probably due to the physical presence of the material on the surgical site. It is expected that the introduction of any material within repaired defects will prolong the inflammatory phase, often delaying blood clot organization and reparative bone neoformation $(44,45)$. However, recent evidence suggests that some proinflammatory cytokines, which orchestrate the cascade of events of inflammation, are necessary for bone repair and that is the persistence of the inflammatory reaction because of infection, which can be harmful and result in an inadequate repair (46-49).

Histometric analysis showed no significant increase in bone neoformation from the second to the fourth week in any of the experimental groups. This finding confirms reports from the literature that "critical or subcritical size" defects in rodent calvaria present a small regeneration rate, which is not completed even in prolonged periods of time $(35,50,51)$.

The rate of bone neoformation was similar in the animals of the Control and Monoolein groups, in both periods analyzed. Monoolein cubic phase gels have been widely used in the pharmaceutical industry as carriers of drugs in various formulations and by different routes of administration (52). Incorporated drugs, including peptides and proteins, undergo slow release, do not lose biological activity (52-55). The rate of bone neoformation in the control groups and monoolein differs from the Monoolein-MMP-2 associated group because of the obvious presence of rhMMP-2 been slowly released, but the presence of exudate in $37 \%$ of the samples in the groups Monoolein and Monoolein+MMP-2 demonstrate the role of inflammation on bone neoformation and, how the presence of a controlled inflammation is beneficial 2 and 4 weeks post-operation.

The results of the present work show that monoolein did not interfere with the repair process (equal results of bone neoformation of the control and monoolein-groups (besides the inflammatory process and appeared to be effective as a carrier of rhMMP-2, corroborating previous literature in which monoolein was tested as a carrier of rhBMP-2 in an acute osteogenic distraction model, in rat mandible (31). The bone repair was also evaluated by histometric method two and four weeks after implantation and the results suggested the efficacy of monoolein to guarantee the release of BMP, which stimulated repair osteogenesis in the treated group (31).

The rate of bone neoformation was significantly higher in the animals of the MMP-2 associated with monoolein group, in both periods analyzed. A critical evidence of the importance of MMP-2 for maintaining normal bone tissue comes from the description of a mutation that inactivates MMP-2, resulting in a severe osteolytic phenotype (56) and the inactivation of MT1-MMP and MM-13 could lead to abnormal bone remodeling which leads to dwarfism $(57,58)$. Experimental models in which the expression of MMP-2 shows that the absence of functional MMP-2 decreases 
the proliferation of osteoblasts $(59,60)$ and, additionally, this enzyme appears to be essential for the formation of osteocyte (60).

The histometric results of the present work show a higher rate of repair in rat calvaria defect in the MMP-2 group, adding evidence to the hypothesis of the importance of the activity of this metalloproteinase for bone formation.

In the pathological processes of inflammation, the MMPs are often found to be MMP-2, -8 and -9 (61), which are associated with the release of cytokines and chemokines, to control the cellular events of inflammation (migration and proliferation and the degradation of the extracellular matrix $(61,62)$. Therefore, some MMPs, including MMP-2, participate in both, the regulation of inflammation and osteolysis associated with inflammatory diseases.

In recent years, a new way of understanding "bone and inflammation" involves the study of chemokines, which are modified by proteases and have their function altered. A classic example that shows the impact of the proteolytic action of MMPs on chemokines is the recruitment of neutrophils in the first hours of the inflammatory response and the completion of this step, followed by the influx of macrophages into the inflammatory site. Some chemokines, when processed primarily by MMP-8, become potent agonists of their respective receptors, which is a critical step in neutrophil recruitment. In contrast, other chemokines are inactivated by macrophages derived MMP-12, which leads to the termination of neutrophil recruitment (63). Thus, the succession of the different cell types in the inflammatory and immune responses stem from the recruitment of these cells, which is determined by chemokines, and these molecules have their function modified by proteases.

Studies on the action of MMP-2 on specific chemokines are quite recent and show that CCL2 (also called MCP-1), a chemokine that plays a central role in recruitment of macrophages, modifies its function when cleaved by MMP-2 (64). In vitro study confirmed that CCL2/MCP-1 cleavage by MMP-2 causes decreased migration of a monocyte lineage (65). In an experimental model of rat paw edema, some MMPs, including MMP-2, showed anti-inflammatory activity by cleaving chemokines, leading to decreased chemotactic activity (66-68).

Considering the potential effects of MMPs on chemokines and their frequent co-localization in inflammatory sites, and also to impose chemokines with bone remodeling, it is possible that cleavage of chemokines contributed to the results observed in the MMP-2 group of the present study.

Several studies have shown that in inflammatory diseases such as periodontitis and rheumatoid arthritis, as well as in osteoporosis, interleukin-1 beta (IL-1 $\beta$ ) has a direct effect on the maturation and differentiation of osteoclasts, stimulating bone absorption $(69,70)$. Some metalloproteinases, including MMP-2, can cleave IL-1 $\beta$ in to biologically inactive fragments, thereby regulating the inflammatory process (71). Inactivation of IL-1 $\beta$ by the high concentration of MMP-2 at the site of the bone defect may also have contributed to the osteostimulatory effect observed in the animals of the MMP-2 group.

Histometric analysis did not show a significant increase in bone neoformation from the second to the fourth week, in none of the experimental groups, confirming reports in the literature that "critical or subcritical" defects in rodent calvaria have a small regeneration rate, which is not completed even in prolonged periods of time. The monoolein alone does not differ from control in the bone repair, but the recruiting inflammatory cells and exudate generated do not benefit the bone repair. But the association with rhMMP-2 has a positive role in bone repair. The authors of the present work concluded that the use of monoolein alone in any surgical intervention on bone is not beneficial, but the association with rhMMP-2 could be beneficial.

The rate of bone neoformation was similar in the Control and monoolein groups and, significantly higher in the animals of the rhMMP-2 associated with monoolein, in both analyzed periods, showing that monoolein had a positive role when associated with MMP-2 on the bone repair and did not interfere with the repair process. Monoolein was effective as a rhMMP-2 carrier at the concentration of $12.5 \mu \mathrm{g}$ of rhMMP-2 bounded to monoolein.

\section{References}

1. Amini AR, Laurencin CT, Nukavarapu SP. Bone tissue engineering: recent advances and challenges. Crit Rev Biomed Engineering 2012; 40 (5): 363-408.

2. Winkler T, Sass FA, Duda GN, Schmidt-Bleek K. A review of biomaterials in bone defect healing, remaining shortcomings and future opportunities for bone tissue engineering: The unsolved challenge. Bone Joint Res 2018; 7 (3): 232-243.

3. Hynes K, Menicanin D, Mrozik K, Gronthos S, Bartold PM. Generation of functional mesenchymal stem cells from different induced pluripotent stem cell lines. Stem Cells Develop 2014; 23 (10): 1084-1096.

4. Machado EG, Issa JP, Figueiredo FA, Santos GR, Galdeano EA, Alves MC et al. A new heterologous fibrin sealant as scaffold to recombinant human bone morphogenetic protein-2 (rhBMP-2) and natural latex proteins for the repair of tibial bone defects. Acta Histochem 2015; 117 (3): 288-296.

5. Ko JY, Park S, Im GI. Osteogenesis from human induced pluripotent stem cells: an in vitro and in vivo comparison with mesenchymal stem cells. Stem cells and development 2014; 23 (15): 1788-1797.

6. Hughes EAB, Grover LM. Characterisation of a novel poly (ether ether ketone)/calcium sulphate composite for bone augmentation. Biomaterials Res 2017; $21: 7$.

7. de Peppo GM, Vunjak-Novakovic G, Marolt D. Cultivation of human bone-like tissue from pluripotent stem cell-derived osteogenic progenitors in perfusion bioreactors. Methods Mol Biol (Clifton, NJ) 2014; 1202: $173-184$.

8. J. CD, Arturo I-F, R. ML, V. JM, J. VE, Mariangeles A et al. Bone Regeneration Mediated by a Bioactive and Biodegradable Extracellular Matrix-Like Hydrogel Based on Elastin-Like Recombinamers. Tissue Engineering Part A 2017; 23 (23-24): 1361-1371.

9. Bilousova G, Jun du H, King KB, De Langhe S, Chick WS, Torchia EC et al. Osteoblasts derived from induced pluripotent stem cells form calcified structures in scaffolds both in vitro and in vivo. Stem Cells (Dayton, Ohio) 2011; 29 (2): 206-216.

10. Issa JPM, Ingraci de Lucia C, dos Santos Kotake BG, Gonçalves Gonzaga M, Tocchini de Figueiredo FA, Mizusaki Iyomasa D et al. The effect of simvastatin treatment on bone repair of femoral fracture in animal model. Growth Factors 2015 2015/03/04; 33 (2): 139-148. 
11. Aline A, Alejandro FP, Sara F, Fellipe ATdF, Maria Cecilia Gorita dos S, Joao Paulo MI. MMPs are Involved in Osteoporosis and are Correlated with Cardiovascular Diseases. Curr Pharm Design 2018; 24: 1-10.

12. Colnot C, Thompson Z, Miclau T, Werb Z, Helms JA. Altered fracture repair in the absence of MMP9. Development 2003; 130 (17): 4123-4133.

13. Almalki SG, Agrawal DK. Effects of matrix metalloproteinases on the fate of mesenchymal stem cells. Stem Cell Res Ther 2016; 7 (1): 129.

14. Souza-Pinto FJ, Moretti AI, Cury V, Marcondes W, Velasco IT, Souza HP. Inducible nitric oxide synthase inhibition increases MMP-2 activity leading to imbalance between extracellular matrix deposition and degradation after polypropylene mesh implant. J Biomed Material Res Part A 2013; 101 (5): 1379-1387.

15. Itoh T, Ikeda T, Gomi H, Nakao S, Suzuki T, Itohara S. Unaltered secretion of beta-amyloid precursor protein in gelatinase $\mathrm{A}$ (matrix metalloproteinase 2)-deficient mice. J Biol Chem 1997; 272 (36): 22389-22392.

16. Ekbote AV, Danda S, Zankl A, Mandal K, Maguire T, Ungerer K. Patient with mutation in the matrix metalloproteinase 2 (MMP2) gene a case report and review of the literature. J Clin Res Pediat Endocrinol 2014; 6 (1): 40-46.

17. Cockett MI, Murphy G, Birch ML, O'Connell JP, Crabbe T, Millican AT et al. Matrix metalloproteinases and metastatic cancer. Biochemical Society Symposium 1998; 63: 295-313.

18. Perez-Amodio S, Beertsen W, Everts V. (Pre-)osteoclasts induce retraction of osteoblasts before their fusion to osteoclasts. J Bone Mineral Res 2004; 19 (10): 1722-1731.

19. Nyman JS, Lynch CC, Perrien DS, Thiolloy S, O'Quinn EC, Patil CA et al. Differential effects between the loss of MMP-2 and MMP-9 on structural and tissue-level properties of bone. J Bone Mineral Res 2011; 26 (6): 1252-1260.

20. Gok F, Crettol LM, Alanay Y, Hacihamdioglu B, Kocaoglu M, Bonafe $\mathbf{L}$ et al. Clinical and radiographic findings in two brothers affected with a novel mutation in matrix metalloproteinase 2 gene. Eur J Pediatr 2010; 169 (3): 363-367.

21. Quintanar-Guerrero D, Buri P. Monoolein: A Review of the Pharmaceutical Applications AU - Ganem-Quintanar, Adriana. Drug Develop Industrial Pharm 2000 2000/01/01; 26 (8): 809-820.

22. D'Antona P, Parker WO, Zanirato MC, Esposito E, Nastruzzi C. Rheologic and NMR characterization of monoglyceride-based formulations. J Biomed Materials Res 2000; 52 (1): 40-52.

23. al Ruhaimi KA. Comparison of different distraction rates in the mandible: an experimental investigation. Internat J Oral Maxillofac Surg 2001; 30 (3): $220-227$.

24. de Albuquerque RF, Jr., Aparecida Del Bel E, Brentegani LG, Moura de Oliveira MT, Mardegan Issa JP. Trigeminal nitric oxide synthase expression correlates with new bone formation during distraction osteogenesis. Calcif Tissue Int 2008; 82 (4): 309-315.

25. Goncalves AN, Meschiari CA, Stetler-Stevenson WG, Nonato MC, Alves CP, Espreafico EM et al. Expression of soluble and functional fulllength human matrix metalloproteinase-2 in Escherichia coli. J Biotechnol 2012; 157 (1): 20-24.

26. Petsch D, Anspach FB. Endotoxin removal from protein solutions. J Biotechnol 2000; 76 (2-3): 97-119.

27. Issa JP, Spadaro AC, Bentley MV, Iyomasa MM, Siessere S, Regalo SC et al. Monoolein and chitosan gels as potential carriers of the rhBMP-2, using decortication surgical technique in Wistar rats as experimental model. Micron (Oxford, England: 1993) 2008; 39 (7): 952-959.

28. Lara MG, Bentley MV, Collett JH. In vitro drug release mechanism and drug loading studies of cubic phase gels. Internat J Pharm 2005; 293 (1-2): 241-520

29. Ellerbroek SM, Wu YI, Stack MS. Type I collagen stabilization of matrix metalloproteinase-2. Arch Biochem Biophys 2001; 390 (1): 51-56.

30. Pereira NT, Issa JP, Nascimento C, Pitol DL, Ervolino E, Cunha MR et al. Effect of alveolex on the bone defects repair stimulated by rhBMP-2: Histomorphometric study. Microsc Res Tech 2012; 75 (1): 36-41.

31. Issa JP, Gonzaga M, Kotake BG, de Lucia C, Ervolino E, Iyomasa M. Bone repair of critical size defects treated with autogenic, allogenic, or xenogenic bone grafts alone or in combination with rhBMP-2. Clin Oral Implants Res 2016; 27 (5): 558-566.

32. Spin-Neto R, de Freitas RM, Pavone C, Cardoso MB, CampanaFilho SP, Marcantonio RA et al. Histological evaluation of chitosan-based biomaterials used for the correction of critical size defects in rat's calvaria. J Biomed Materials Res Part A 2010; 93 (1): 107-114.

33. Hijova E. Matrix metalloproteinases: their biological functions and clinical implications. Bratisl Lek Listy 2005; 106 (3): 127-132.

34. Liska V, Sutnar A, Holubec L, Jr., Vrzalova J, Treska V, Skalicky T et al. Matrix metalloproteinases and their inhibitors in correlation to proliferative and classical tumour markers during surgical therapy of colorectal liver metastases. Bratisl Lek Listy 2012; 113 (2): 108-113.

35. Cooper GM, Mooney MP, Gosain AK, Campbell PG, Losee JE, Huard J. Testing the critical size in calvarial bone defects: revisiting the concept of a critical-size defect. Plast Reconstr Surg 2010; 125 (6): $1685-1692$.

36. Gomes PS, Fernandes MH. Rodent models in bone-related research: the relevance of calvarial defects in the assessment of bone regeneration strategies. Laboratory Animals 2011; 45 (1): 14-24.

37. Schmitz JP, Hollinger JO. The critical size defect as an experimental model for craniomandibulofacial nonunions. Clin Orthop Related Res 1986 (205): 299-308.

38. Gosain AK, Song L, Yu P, Mehrara BJ, Maeda CY, Gold LI et al. Osteogenesis in cranial defects: reassessment of the concept of critical size and the expression of TGF-beta isoforms. Plast Reconstr Surg 2000; 106 (2): 360-371.

39. Yang J-W, Park H-J, Yoo K-H, Chung K, Jung S, Oh H-K et al. A comparison study between periosteum and resorbable collagen membrane on iliac block bone graft resorption in the rabbit calvarium. Head Face Med 2014; 10 (1): 15.

40. Li Z, Pan J, Ma J, Zhang Z, Bai Y. Microarray gene expression of periosteum in spontaneous bone regeneration of mandibular segmental defects. Sci Rep 2017 2017/10/19; 7 (1): 13535.

41. Silva JPN, Oliveira IMSC, Oliveira ACN, Lúcio M, Gomes AC, Coutinho PJG et al. Structural dynamics and physicochemical properties of pDNA/DODAB:MO lipoplexes: Effect of $\mathrm{pH}$ and anionic lipids in inverted non-lamellar phases versus lamellar phases. Biochim Biophys Acta - Biomembranes 2014 2014/10/01/; 1838 (10): 2555-2567.

42. Liu H, Wang Z, Li Y, Li W, Chen Y. Simvastatin prevents proliferation and bone metastases of lung adenocarcinoma in vitro and in vivo. Neoplasma 2013; 60 (3): 240-246.

43. Pasieka Z, Stepien H, Czyz W, Pomorski L, Kuzdak K. Concentration of metalloproteinase-2 and tissue inhibitor of metalloproteinase-2 in 
the serum of patients with benign and malignant thyroid tumours treated surgically. Endocr Regul 2004; 38 (2): 57-63.

44. Okamoto T, Okamoto R, Alves Rezende MC, Gabrielli MF. Interference of the blood clot on granulation tissue formation after tooth extraction. Histomorphological study in rats. Brazil Dent J 1994; 5 (2): 85-92.

45. Pinheiro AL, Limeira Junior Fde A, Gerbi ME, Ramalho LM, Marzola C, Ponzi EA. Effect of low level laser therapy on the repair of bone defects grafted with inorganic bovine bone. Brazil Dent J 2003; 14 (3): $177-181$.

46. Thomas MV, Puleo DA. Infection, inflammation, and bone regeneration: a paradoxical relationship. J Dent Res 2011; 90 (9): 1052-1061.

47. Waters RV, Gamradt SC, Asnis P, Vickery BH, Avnur Z, Hill E et al. Systemic corticosteroids inhibit bone healing in a rabbit ulnar osteotomy model. Acta Orthop Scand 2000; 71 (3): 316-321.

48. O'Keefe RJ, Mao J. Bone tissue engineering and regeneration: from discovery to the clinic - an overview. Tissue Engineer Part B, Rev 2011; 17 (6): 389-392.

49. Loi F, Córdova LA, Pajarinen J, Lin T-h, Yao Z, Goodman SB. Inflammation, fracture and bone repair. Bone 2016; 86: 119-130.

50. Fernandes RA, Christofaro DG, Casonatto J, Codogno JS, Rodrigues EQ, Cardoso ML et al. Prevalence of dyslipidemia in individuals physically active during childhood, adolescence and adult age. Arq Bras Cardiol 2011 10/01; 97 (4): 317-323.

51. Porto GG, Vasconcelos BCdE, Andrade ESdS, Carneiro SCdAS, Frota MSM. Is a $5 \mathrm{~mm}$ rat calvarium defect really critical? Acta Cir Brasil 2012; 27: 757-760.

52. Shah MH, Paradkar A. Cubic liquid crystalline glyceryl monooleate matrices for oral delivery of enzyme. Internat J Pharm 2005; 294 (1-2): 161-171.

53. Lee J, Kellaway IW. In vitro peptide release from liquid crystalline buccal delivery systems. Internat J Pharm 2000; 195 (1-2): 29-33.

54. Lee J, Kellaway IW. Buccal permeation of [D-Ala (2), D-Leu (5)] enkephalin from liquid crystalline phases of glyceryl monooleate. Internat J Pharm 2000; 195 (1-2): 35-38.

55. Shah JC, Sadhale Y, Chilukuri DM. Cubic phase gels as drug delivery systems. Adv Drug Delivery Rev 2001 2001/04/25/; 47 (2): 229-250.

56. Martignetti JA, Aqeel AA, Sewairi WA, Boumah CE, Kambouris M, Mayouf SA et al. Mutation of the matrix metalloproteinase 2 gene (MMP2) causes a multicentric osteolysis and arthritis syndrome. Nature Genetics 2001; 28 (3): 261-265.

57. Holmbeck K, Bianco P, Caterina J, Yamada S, Kromer M, Kuznetsov SA et al. MT1-MMP-deficient mice develop dwarfism, osteopenia, arthritis, and connective tissue disease due to inadequate collagen turnover. Cell 1999; 99 (1): 81-92.

58. Nakatani T, Chen T, Partridge NC. MMP-13 is one of the critical mediators of the effect of HDAC4 deletion on the skeleton. Bone 2016; 90: $142-151$.
59. Mosig RA, Dowling O, DiFeo A, Ramirez MC, Parker IC, Abe E et al. Loss of MMP-2 disrupts skeletal and craniofacial development and results in decreased bone mineralization, joint erosion and defects in osteoblast and osteoclast growth. Human Mol Genet 2007; 16 (9): 1113-1123.

60. Inoue K, Mikuni-Takagaki Y, Oikawa K, Itoh T, Inada M, Noguchi $\mathbf{T}$ et al. A crucial role for matrix metalloproteinase 2 in osteocytic canalicular formation and bone metabolism. Journal Biol Chem 2006; 281 (44): 33814-33824.

61. Chow AK, Cena J, Schulz R. Acute actions and novel targets of matrix metalloproteinases in the heart and vasculature. Brit J Pharm 2007; 152 (2): 189-205.

62. Galis ZS, Khatri JJ. Matrix metalloproteinases in vascular remodeling and atherogenesis: the good, the bad, and the ugly. Circulat Res 2002; 90 (3): 251-262.

63. Starr AE, Dufour A, Maier J, Overall CM. Biochemical analysis of matrix metalloproteinase activation of chemokines CCL15 and CCL23 and increased glycosaminoglycan binding of CCL16. J Biol Chem 2012; 287 (8): 5848-5860.

64. Qian BZ, Li J, Zhang H, Kitamura T, Zhang J, Campion LR et al. CCL2 recruits inflammatory monocytes to facilitate breast-tumour metastasis. Nature 2011; 475 (7355): 222-225.

65. Denney H, Clench MR, Woodroofe MN. Cleavage of chemokines CCL2 and CXCL10 by matrix metalloproteinases-2 and -9: implications for chemotaxis. Biochem Biophys Res Comm 2009; 382 (2): 341-347.

66. McQuibban A, Butler G, H Gong J, Bendall L, Power C, ClarkLewis I et al. Matrix Metalloproteinase Activity Inactivates the CXC Chemokine Stromal Cell-derived Factor-12001. 43503-43508 p.

67. McQuibban GA, Gong JH, Tam EM, McCulloch CA, Clark-Lewis I, Overall CM. Inflammation dampened by gelatinase A cleavage of monocyte chemoattractant protein-3. Science (New York, NY) 2000; 289 (5482): 1202-1206.

68. McQuibban GA, Gong JH, Wong JP, Wallace JL, Clark-Lewis I, Overall CM. Matrix metalloproteinase processing of monocyte chemoattractant proteins generates CC chemokine receptor antagonists with anti-inflammatory properties in vivo. Blood 2002; 100 (4): 1160-1167.

69. Bataille R, Barlogie B, Lu ZY, Rossi JF, Lavabre-Bertrand T, Beck T et al. Biologic effects of anti-interleukin- 6 murine monoclonal antibody in advanced multiple myeloma. Blood 1995; 86 (2): 685-691.

70. Mercado FB, Marshall RI, Klestov AC, Bartold PM. Relationship between rheumatoid arthritis and periodontitis. J Periodontol 2001; 72 (6): 779-787.

71. Ito A, Mukaiyama A, Itoh Y, Nagase H, Thogersen IB, Enghild JJ et al. Degradation of interleukin 1 beta by matrix metalloproteinases. J Biol Chem 1996; 271 (25): 14657-14660.

Received March 31, 2020. Accepted April 6, 2020. 